Chin. J. Astron. Astrophys. Vol.0 (200x) No.0, 000-000

(http://www.chjaa.org )

Chinese Journal of

Astronomy and

Astrophysics

\title{
Fitting Formulae for the Effects of Binary Interactions on Lick Indices and Colours of Stellar Populations
}

\author{
Zhong-Mu $\mathrm{Li}^{1}{ }^{\star}$ and Zhan-Wen $\mathrm{Han}^{2}$ \\ 1 Dali Uinversity, Dali, 671003, China \\ 2 National Astronomical Observatories/Yunnan Observatory, the Chinese Academy of \\ Sciences, Kunming, 650011, China
}

Received 2007 Dec. 17; accepted 2001 month day

\begin{abstract}
More than about 50\% stars are in binaries, but most stellar population studies take single star stellar population (ssSSP) models, which do not take binary interactions into account. In fact, the integrated peculiarities of ssSSPs are various from those of stellar populations with binary interactions (bsSSPs). Therefore, it is necessary to investigate the effects of binary interactions on the Lick indices and colours of populations detailedly. We show some formulae for calculating the difference between the Lick indices and colours of bsSSPs, and those of ssSSPs. Twenty-five Lick indices and 12 colours are studied in the work. The results can be conveniently used for calculating the effects of binary interactions on stellar population studies and for adding the effects of binary interactions into present ssSSP models. The electrical data and fortran procedures of the paper can be obtained on request to the authors.
\end{abstract}

Key words: galaxies: stellar content — galaxies: elliptical and lenticular, cD

\section{INTRODUCTION}

In the golden era for studying the formation and evolution of galaxies, evolutionary stellar population synthesis has been an important technique for such works, as some stellar characteristics (e.g., stellar age and metallicity) of galaxies can be determined via this technique. Many stellar population synthesis models, e.g., Worthey 1994, Buzzoni (1995), Bressan et al. (2003), Vázquez \& Leitherer (2005), $\quad$ Bruzual \& Charlot (2003)| |Fioc \& Rocca-Volmerange (1997) Vazdekis et al. (2003), Delgado et al. (2005), and Zhang et al. (2005), were brought forward and have been widely used for stellar population studies. However, the above models except the one of Zhang et al. (2005) are single star stellar population (ssSSP) models that did not take the effects of binary interactions into account. According to the results of Han et al. (2001). more than $50 \%$ stars of the Galaxy are in binaries and binaries evolve differently from single

$\star$ E-mail: zhongmu.li@gmail.com 
stars. The real stellar populations of galaxies and star clusters consist of not only single stars, but also binary stars. Thus ssSSPs are different from the real populations of galaxies and star clusters. In fact, binary evolution can affect the integrated peculiarities (e.g., the spectral energy distributions in UV bands) of stellar populations significantly (see, e.g., Han et al. 2007). Therefore, the effects of binary evolution should be taken into account when modeling the stellar populations of galaxies and star clusters.

A few works have been tried to investigate the effects of binary evolution on stellar population synthesis. For example, Zhang et al. (2005) tried to model populations via binary stars. In addition, Li \& Han (2008b) built an isochrone database for quickly modeling binary star stellar populations (bsSSPs) and a rapid model (hereafter $R P S$ model) for both ssSSPs and bsSSPs. In special, Li \& Han (2008c) investigated the detailed effects of binary interactions on the results of stellar population synthesis and the results of stellar population studies. The results can help us to understand how the results obtained via ssSSPs are different from those obtained via bsSSPs, when taking $\mathrm{H} \beta-[\mathrm{MgFe}]$ (Thomas et al. 2003) and two-colour methods. According to the results of $\mathrm{Li} \& \mathrm{Han}(2008 \mathrm{c})$, when we use ssSSP models to measure the stellar ages and metallicities of galaxies, we will obtain obviously younger ages or lower metallicities compared to the real values of populations, using $\mathrm{H} \beta-[\mathrm{MgFe}]$ and two-colour methods, respectively. However, there is no clear relation between the real metallicities and fitted (via ssSSPs) results of populations. One please refer to Li \& Han (2008c) for more details. In this case, it is difficult to get more accurate information about the stellar metallicities of galaxies via ssSSP models, and then the chemical evolution of galaxies. Furthermore, the previous work only shows the results for $\mathrm{H} \beta-[\mathrm{MgFe}]$ method, when taking Lick indices for works, but some other methods and indices are also used in investigations. Thus it is necessary to investigate the effects of binary interactions on the results of stellar population studies obtained via various Lick indices further. The metallicity range of above bsSSP models (Zhang et al. (2005), Li \& Han (2008b)) seems not wide enough (see Li et al. 2006), as it only covers the metallicity range poorer than $0.03(Z \leq 0.03)$. This is limited by the star evolution code. If we can give the relation between the effects of binary interactions and the stellar-population parameters (age and metallicity), we will be able to understand the populations of galaxies and star clusters further, and more detailed investigations about galaxy formation and evolution will have in the future. Therefore, it is valuable to study how the effects of binary interactions on integrated peculiarities of populations change with stellar age and metallicity. We have a try in this work. As a result, a few formulae for describing the relations between the effects of binary interactions on integrated indices (Lick indices and colours indices) and stellar-population parameters are presented.

The structure of the paper is as follows. In Sect. 2 we introduce the stellar population model used in the paper. In Sect. 3 we show the fitting formulae for the changes of 25 Lick indices caused by binary interactions when comparing to those of ssSSPs. In Sect. 4 we give similar investigations to 12 colours of populations. Finally, we give our discussion and conclusion in Sect. 5 .

\section{STELLAR POPULATION MODEL USED IN THE PAPER}

The RPS model of Li \& Han (2008b) is used in this investigation, because there is no more suitable model. The model calculated the integrated peculiarities $(0.3 \AA$ SEDs, Lick indices and colours) of both bsSSPs and ssSSPs with two widely used initial mass functions (IMFs) (Salpeter and Chabrier IMFs). Each bsSSP contains about 50\% stars that are in binaries with orbital periods less than $100 \mathrm{yr}$ (the typical value of the Galaxy, see Han et al. 1995). Binary interactions such as mass transfer, mass accretion, common-envelope evolution, collisions, supernova kicks, angular momentum loss mechanism, and tidal interactions are considered when evolving binaries via the rapid stellar evolution code of Hurley et al. (2002). Therefore, the RPS model 
is suitable for studying the effects of binary interactions on stellar population synthesis. The details about the model can be seen in the paper of Li \& Han (2008b) and Li \& Han (2008c). For convenience, we take stellar populations with Salpeter IMF for our standard investigations in the work, but the results obtained via populations with Chabrier IMF are also presented.

\section{FITTING FORMULAE FOR THE EFFECTS OF BINARY INTERACTIONS ON 25 LICK INDICES}

Lick indices are the most widely used indices in stellar population studies, because they can disentangle the well-known stellar age-metallicity degeneracy (Worthey 1994). Making use of an age-sensitive index (e.g., $\mathrm{H} \beta$ ) together with a metallicity-sensitive index (e.g., [MgFe], see Thomas et al. 2003), the stellar age and metallicity of a population can be determined. Thus to investigate the effects of binary interactions on the Lick indices of stellar populations is important. The work of $\mathrm{Li} \& \mathrm{Han}$ (2008c) showed that binary interactions make the $\mathrm{H} \beta$ index less while some metal-line indices larger compared to those of ssSSPs. It leads to younger age estimate when we take ssSSPs for works. However, in that work, only the results obtained via $\mathrm{H} \beta-[\mathrm{MgFe}]$ method are compared to the real values of populations. Some other Lick indices, e.g., $\mathrm{Mg} 2, \mathrm{H} \delta_{\mathrm{A}}$, and $\mathrm{H} \gamma_{\mathrm{A}}$, are also widely used in studies (e.g., Gallazzi et al. 2005). Therefore, it is necessary to study the effects of binary interactions on more Lick indices and give the quantitative relations between binary effects and stellar-population parameters. Here we study on 25 widely used indices and fit the relations between the changes caused by binary interactions and the stellar-population parameters (age and metallicity), via a polynomial fitting method. The results can be used to calculate the differences between the 25 Lick indices of two kinds of populations with small errors (typically less than $0.03 \AA$ or mag). All Lick indices are on the Lick system (see, e.g., Worthey 1994). When comparing to ssSSPs, the effects of binary interactions on Lick indices can be calculated from stellar age and metallicity, by

$$
\Delta I=\sum_{i=1}^{5}\left(\mathrm{C}_{i 1}+\mathrm{C}_{i 2} Z+\mathrm{C}_{i 3} Z^{2}\right) t^{i-1},
$$

where $\Delta I$ is the change of a Lick index caused by binary interactions, and $Z$ is stellar metallicity, while $t$ is stellar age. The detailed coefficients for our standard investigation are shown in Tables 1 and 2. Those for populations with Chabrier IMF are shown in the Appendix. For clearly, in Figs. 1, 2, and 3, we compare the changes calculated by equation (1) with the original values obtained in the work. Note that we only show the fittings for 12 widely used Lick indices here, because the fittings for other indices are similar. As we see, for the indices shown, the values calculated by the above equation are consistent with those obtained directly by comparing the Lick indices of bsSSPs and ssSSPs, with typical errors of $0.03 \AA$ or mag. Therefore, the fitting formulae presented can be used to calculate the differences of Lick indices of bsSSPs and ssSSPs, using the age and metallicity of populations. In addition, the results show that binary interactions make age-sensitive indices (e.g., $\mathrm{H} \beta, H \delta_{\mathrm{A}}, H \delta_{\mathrm{F}}, H \gamma_{\mathrm{A}}, H \gamma_{\mathrm{F}}$ ) of a bsSSP larger than those of its corresponding (with the same age and metallicity) ssSSP, while making metallicitysensitive indices (e.g., $\mathrm{Mg}$ or Fe indices) of a bsSSP less than that of its corresponding ssSSP. This is similar to that shown in the paper of Li \& Han (2008c). Furthermore, it is shown that the differences between Lick indices of bsSSPs and ssSSPs increase with age when stellar age is small ( $<$ about $2.5 \mathrm{Gyr}$ ), and they decrease with age for larger age. This results from the star sample (i.e., the fraction of binaries and the relation between the masses of the two components of each binary) of stellar populations. As a whole, the values calculated via the fitting formulae obtained by the paper reproduce the evolution of the difference between Lick indices of bsSSPs and ssSSPs. 

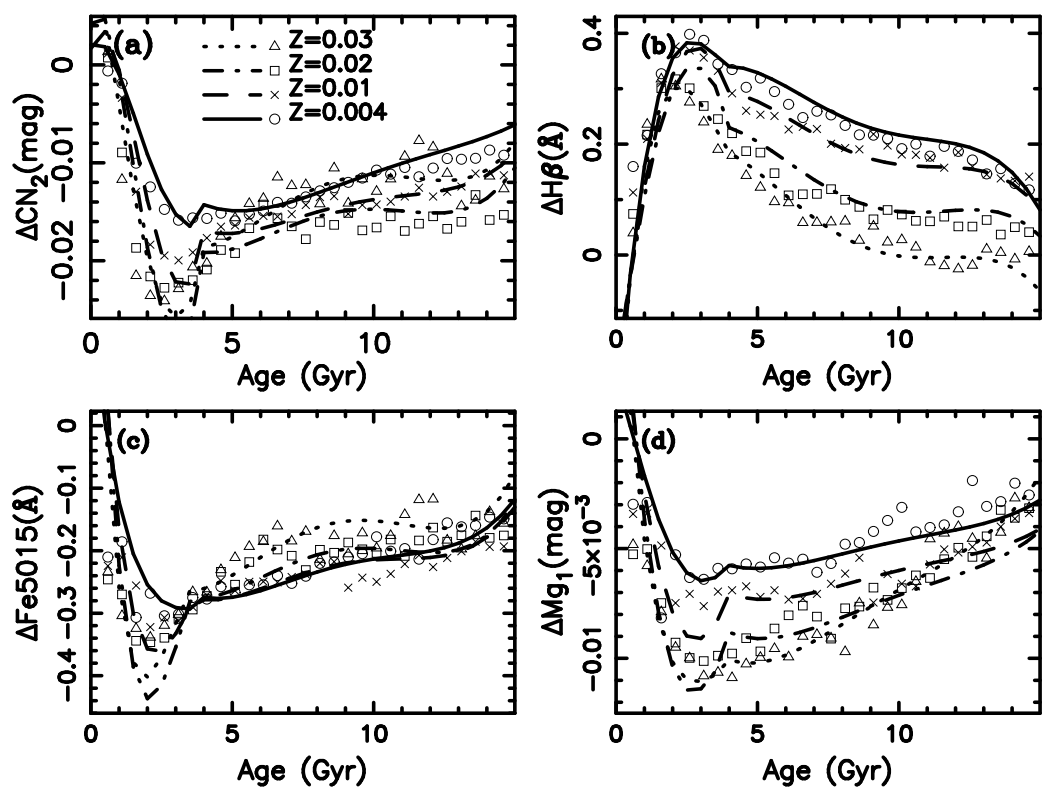

Fig. 1 Comparison of fitted and original values for the effects of binary interactions on four Lick indices. Circles, crosses, squares, and triangles are for the metallicities of $Z$ $=0.004,0.01,0.02$, and 0.03 , respectively. Solid, dashed, dash-dotted, and dotted lines show the fittings for the above four metallicities, respectively. The values of y-axes are calculated by subtracting the Lick indices of a bsSSP from that of its corresponding (with the same age and metallicity) ssSSP. Panels a), b), c), and d) are for $\mathrm{CN}_{2}, \mathrm{H} \beta$, $\mathrm{Fe} 5015$, and $\mathrm{Mg}_{1}$, respectively.

\section{FITTING FORMULAE FOR EFFECTS OF BINARY INTERACTIONS ON 12 COLOURS}

Because colours can also be used for stellar population studies, we fit the formulae for calculating the colour changes cased by binary interactions when comparing to ssSSPs. One can refer to, e.g., Li et al. (2007), Li \& Han (2007), Li \& Han (2008a), Li \& Han (2008c), for the application of colours in stellar population studies. Some Johnson system colours, Sloan Digital Sky Survey system (hereafter SDSS-ugriz system) colours, and some composite colours that consist of both Johnson and an SDSS-ugriz magnitudes are studied. We only study the colours of populations with $Z \geq 0.004$, because it is difficult to determine the stellar age and metallicity of metalpoor (e.g., $Z<0.008$ ) populations via colours under the typical observational uncertainties (Li \& Han (2008a)) and metallicity affect the colours of metal-poor populations stronger. Thus one should use the results shown here for more metal-poor populations carefully. Because it is impossible to give the formulae for all colours, we give some ones for calculating the effects of binary interactions on 12 important colours, which are sensitive to stellar age or metallicity, according to the work of $\mathrm{Li} \& \mathrm{Han}(2008 \mathrm{a})$. The 12 colours are $(B-V),(V-K),(I-H)$, $(R-K),(B-K),(I-K),(u-r),(r-K),(u-R),(u-K),(z-K)$, and $(g-J)$. Note

\footnotetext{
${ }^{1}$ Colours $(r-K),(u-R),(u-K),(z-K)$, and $(g-J)$ are composite colours. The $U B V R I J H K$ magnitudes are on Johnson system, and ugriz magnitudes on SDSS-ugriz system.
} 

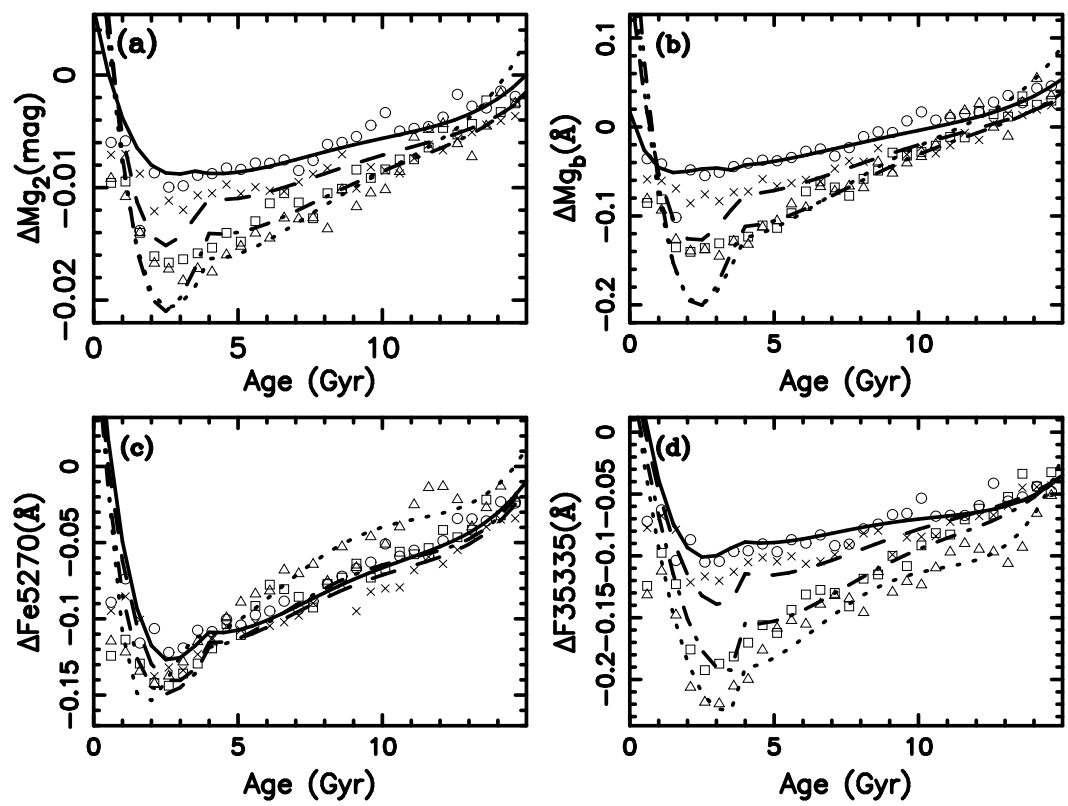

Fig. 2 Similar to Fig. 1, but for $\mathrm{Mg}_{2}, \mathrm{Mg}_{\mathrm{b}}$, Fe5270, and Fe5335.
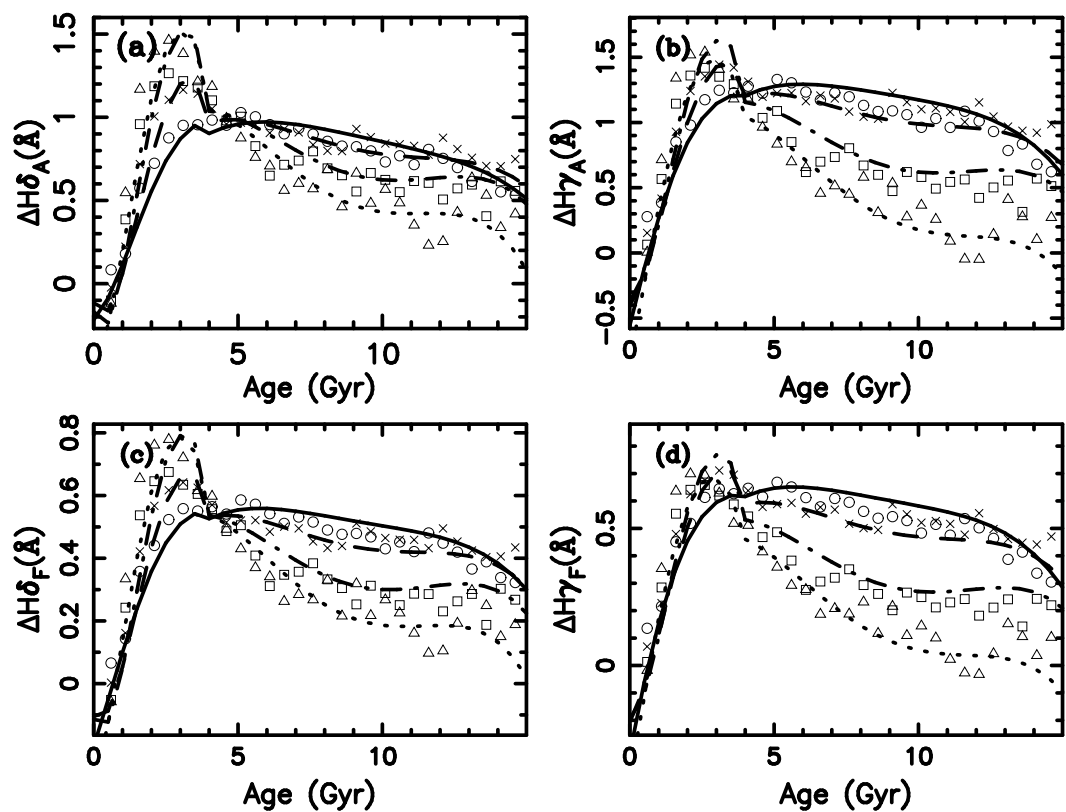

Fig. 3 Similar to Fig. 1, but for $\mathrm{H} \delta_{\mathrm{A}}, \mathrm{H} \gamma_{\mathrm{A}}, \mathrm{H} \delta_{\mathrm{F}}$, and $\mathrm{H} \gamma_{\mathrm{F}}$. 

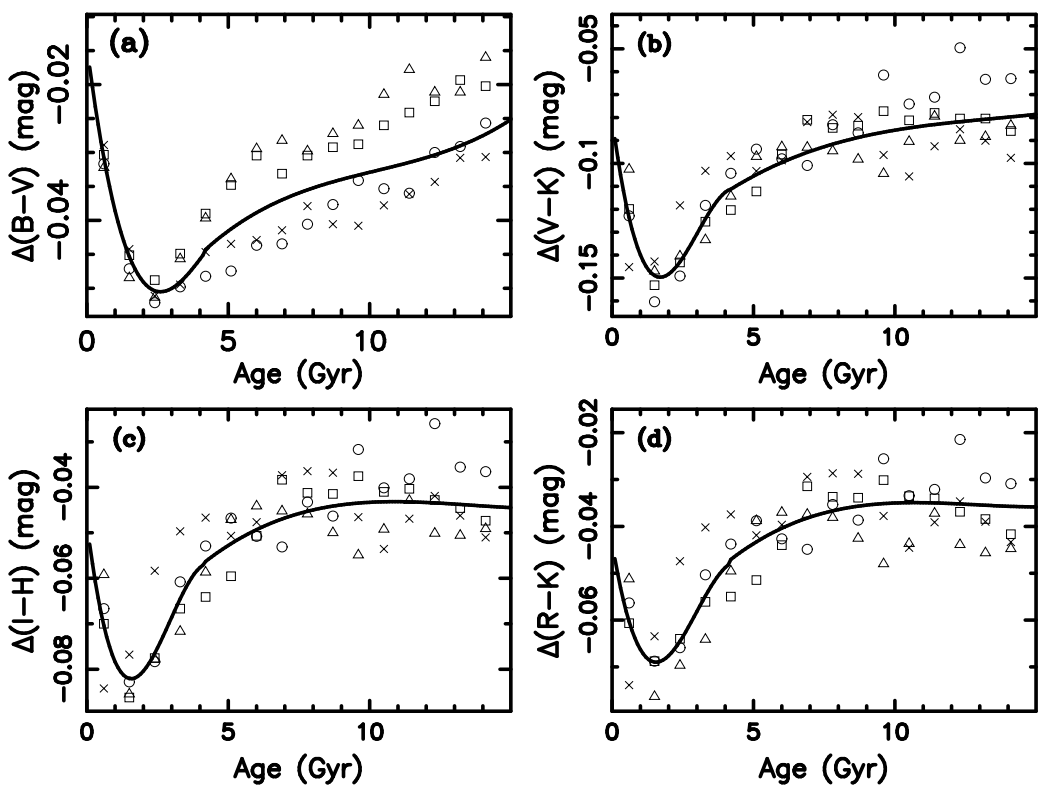

Fig. 4 Fittings for the effects of binary interactions on four colours of populations. Circles, crosses, squares, and triangles show the values obtained directly from comparing the colours of bsSSPs and ssSSPs and are for metallicities of 0.004, 0.01, 0.02, and 0.03 , respectively. Solid lines show the fittings. The y-axis is obtained by subtracting the colour of a bsSSP from that of an ssSSP (with the same age and metallicity as the bsSSP). The four panels are for $(B-V),(V-K),(I-H)$, and $(R-K)$, respectively.

that $(B-V),(u-r),(u-R)$, and $(z-K)$ are more sensitive to stellar age and the others to metallicity. Our work shows that the changes of the above colours caused by binary interactions can be expressed as

$$
\Delta I^{\prime}=\sum_{i=1}^{4} \mathrm{C}_{i} t^{i-1}
$$

where $\Delta I^{\prime}$ is the change of colours caused by binary interactions, and $t$ is stellar age. The coefficients of the equation are shown in Table 3. Note that the results for populations with both Salpeter IMF (standard investigation) and Chabrier IMF are listed in the table. We can find that equation (2) does not include the metallicity of populations. The reason is that colours are less sensitive to metallicity compared to Lick indices and they seem to be affected by the Mento Carlo method used to generate our star sample. The fitting of the effects of binary interactions on 12 colours are shown in Figs. 4, 5, and 6. As we see, the fitting formulae can give average colour changes caused by binary interactions. However, because the results calculated using equation (2) have typical errors about $0.02 \mathrm{mag}$, some additional uncertainties may be brought into the results of stellar population studies. 

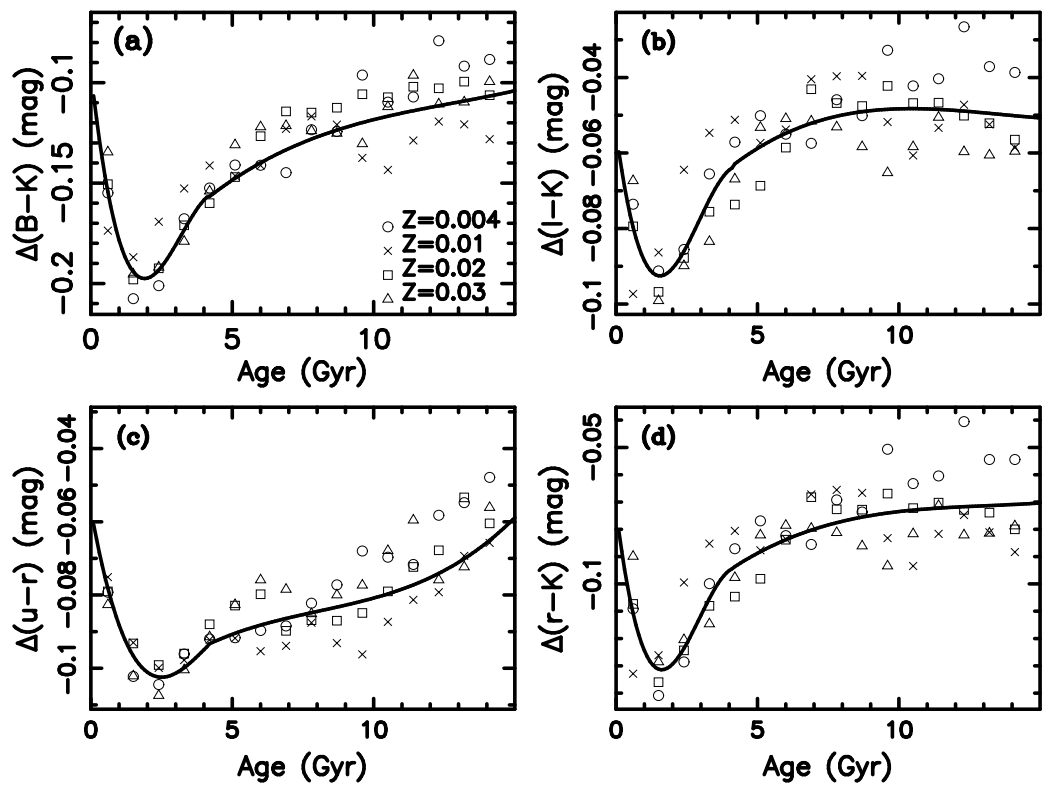

Fig. 5 Similar to Fig. 4, but for $(B-K),(I-K),(u-r)$, and $(r-K)$.
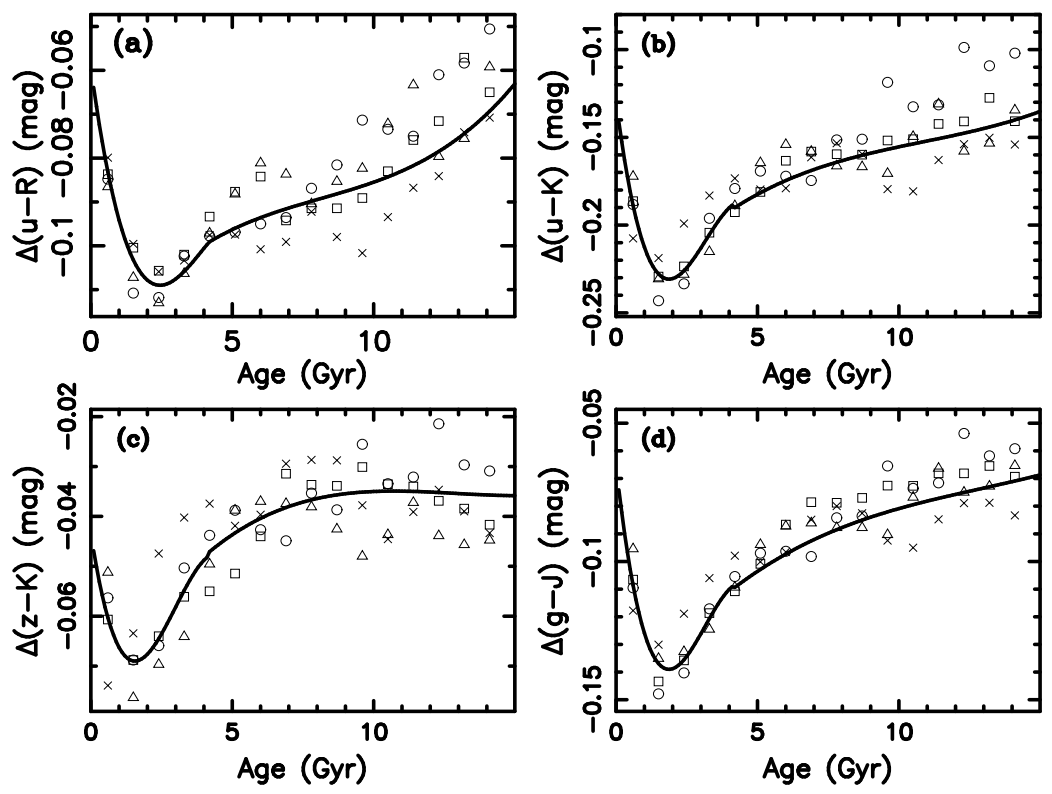

Fig. 6 Similar to Fig. 4 , but for $(u-R),(u-K),(z-K)$, and $(g-J)$. 


\section{DISCUSSION AND CONCLUSIONS}

We present some formulae for conveniently computing the changes caused by binary interactions in 25 Lick indices and 12 colours, compared to the indices of single star stellar populations (ssSSPs). It is shown that the fitting formulae presented in the paper can reproduce the changes in Lick indices caused by binary interactions with small errors and can be used to estimate similar changes in colours. It is also found that binary interactions make age-sensitive Lick indices (not only $\mathrm{H} \beta$, but also $H \delta_{\mathrm{A}}, H \delta_{\mathrm{F}}, H \gamma_{\mathrm{A}}, H \gamma_{\mathrm{F}}$ ) larger, while making metallicity-sensitive indices less compared to those of ssSSPs. This is useful for estimating the effects of binary evolution on the results of stellar population studies and for adding the effects of binary interactions into ssSSP models. Therefore, when an age-sensitive Lick index is used together with a metallicitysensitive Lick index to determine the ages and metallicities of populations, younger ages will be obtained, especially for metal-poor populations, see also Li \& Han (2008c). Note that only binary star stellar populations (bsSSPs) and ssSSPs with four metallicities $(Z=0.004,0.01$, 0.02 , and 0.03 ) are used in the work. This is actually limited by the metallicity coverage of stellar population model and the less sensitivities of colours to metallicity. Thus the results are more suitable for studying metal-rich $(Z \geq 0.004)$ populations, because the differences between integrated peculiarities of populations with various metallicities seem larger for metalpoor populations. In addition, although different formulae are presented for populations with various initial mass functions (IMFs), the changes calculated via two kinds of formulae (the formulae for populations with Salpeter and Chabrier IMFs) are similar. In other words, the changes calculated by the formulae obtained using populations with Salpeter IMF or Chabrier IMF can give us some pictures for the effects of binary interactions. Furthermore, because the Monte Carlo technique used to generate the binary sample of stellar populations make the evolution of integrated peculiarities of populations unsmooth, some results, especially, those for colours, may be somewhat rough. The additional uncertainties involved should be taken into account. If possible, we will give more detailed studies in the future.

Acknowledgements We thank the referee, Profs. Gang Zhao, Xu Zhou, Licai Deng, Xu Kong, Tinggui Wang, and Li Zhang for useful suggestions or discussions. This work is supported by the Chinese National Science Foundation (Grant Nos. 10433030, 10521001, 2007CB815406).

\section{References}

Bressan A., Chiosi, C., Fagotto F., 1994, ApJS, 94, 63

Bruzual G., Charlot S., 2003, MNRAS, 344, 1000

Buzzoni, A., 1995, ApJS, 98, 69

Delgado R. M. G., Cerviño M., Martins L. P. et al., 2005, MNRAS, 357, 945

Fioc M., Rocca-Volmerange, B., 1997, A\&A, 326, 950

Gallazzi A., Charlot S., Brinchmann J. et al., 2005, MNRAS, 362, 41

Han Z., Eggleton P. P., Podsiadlowski P. et al., 2001, Progress in Astronomy, 19, 242

Han Z., Podsiadlowski P., Eggleton P. P., 1995, MNRAS, 272, 800

Han Z., Podsiadlowski P., Lynas-Gray A. E., 2007, MNRAS, 380, 1098

Hurley J. R., Tout C. A., Pols O. R., 2002, MNRAS, 329, 897

Li Z., Zhang F., Han Z., 2006, ChJAA, 6, 669

Li Z., Han Z., Zhang F., 2007, A\&A, 464, 853

Li Z., Han Z., 2007, A\&A, 471, 795

Li Z., Han Z., 2008a, MNRAS, 385, 1270

Li Z., Han Z., 2008b, MNRAS, 387, 105 
Li Z., Han Z., 2008c, ApJ, 685, 225

Thomas D., Maraston C., Bender R., 2003, MNRAS, 343, 279

Vazdekis A., Cenarro A. J., Gorgas J. et al., 2003, MNRAS, 340, 1317

Vázquez G. A., Leitherer C., 2005, ApJ, 621, 695

Worthey G., 1994, ApJS, 95, 107

Worthey G., Faber S. M., Gonzalez J. J. et al., 1994, ApJS, 94, 687

Zhang F., Li L., Han Z., 2005, MNRAS, 364, 503

This paper was prepared with the ChJAA LATEX macro v1.0. 
Appendix A: COEFFICIENTS FOR CALCULATING THE EFFECTS OF BINARY INTERACTIONS ON 25 LICK INDICES OF

POPULATIONS WITH CHABRIER IMF 
Table 1 Coefficients for equation (1). The coefficients are obtained via stellar populations with Salpeter IMF and can be used for populations younger than 4 Gyr (Age $<4 \mathrm{Gyr})$.

\begin{tabular}{|c|c|c|c|c|c|c|}
\hline Index & $j$ & $\mathrm{C}_{1 \mathrm{j}}$ & $\mathrm{C}_{2 \mathrm{j}}$ & $\mathrm{C}_{3 \mathrm{j}}$ & $\mathrm{C}_{4 \mathrm{j}}$ & $\mathrm{C}_{5 \mathrm{j}}$ \\
\hline \multirow{3}{*}{$\mathrm{CN}_{1}$} & 1 & 0.0061004 & -0.0124136 & 0.0044131 & -0.0010174 & 0.0000862 \\
\hline & 2 & -0.7411409 & 3.6764976 & -3.4540761 & 0.9481077 & -0.0789850 \\
\hline & 3 & 30.8122734 & -119.0088796 & 98.2842545 & -25.5632396 & 2.0732871 \\
\hline \multirow{3}{*}{$\mathrm{CN}_{2}$} & 1 & 0.0031344 & -0.0060648 & 0.0018661 & -0.0005851 & 0.0000607 \\
\hline & 2 & -0.3472402 & 2.6820361 & -2.8891002 & 0.8270163 & -0.0703722 \\
\hline & 3 & 20.0763558 & -92.9530724 & 84.0072412 & -22.6637506 & 1.8767287 \\
\hline \multirow{3}{*}{$\mathrm{Ca} 4227$} & 1 & 0.0116927 & -0.0652076 & 0.0542984 & -0.0142708 & 0.0011505 \\
\hline & 2 & 2.6086437 & 1.4449921 & -5.0762513 & 1.6986709 & -0.1529064 \\
\hline & 3 & -71.6010754 & 65.3934655 & -2.6926390 & -6.3483260 & 0.8599647 \\
\hline \multirow{3}{*}{ G4300 } & 1 & 0.5779073 & -1.3565672 & 0.6261188 & -0.1240817 & 0.0083961 \\
\hline & 2 & -50.8998137 & 134.5958812 & -94.7989188 & 23.2438808 & -1.8011864 \\
\hline & 3 & 1740.9893518 & -4249.3881941 & 2828.2830757 & -668.7058103 & 50.9809128 \\
\hline \multirow{3}{*}{ Fe4383 } & 1 & 0.1013725 & -0.1526486 & -0.0164836 & 0.0124241 & -0.0013244 \\
\hline & 2 & 11.0894652 & -9.0004221 & -12.0126956 & 4.9108205 & -0.4569935 \\
\hline & 3 & -75.5186140 & -252.7173536 & 549.1707675 & -171.1008580 & 14.7310943 \\
\hline \multirow{3}{*}{$\mathrm{Ca} 4455$} & 1 & -0.0047432 & 0.0186972 & -0.0197387 & 0.0040785 & -0.0002709 \\
\hline & 2 & 6.5346797 & -11.0259844 & 2.2737411 & 0.1733499 & -0.0467838 \\
\hline & 3 & -98.3181765 & 98.4157661 & 49.1921063 & -27.0189711 & 2.8005540 \\
\hline \multirow{3}{*}{ Fe4531 } & 1 & 0.0550772 & -0.1274444 & 0.0222163 & -0.0009561 & -0.0000671 \\
\hline & 2 & 18.2809537 & -32.2387726 & 11.1472063 & -1.0492667 & -0.0001559 \\
\hline & 3 & -313.2684866 & 374.3927838 & -24.2302367 & -31.1448033 & 4.4257825 \\
\hline \multirow{3}{*}{$\mathrm{Fe} 4668$} & 1 & -0.0717857 & 0.2203558 & -0.1810129 & 0.0435325 & -0.0033410 \\
\hline & 2 & 27.2930010 & -66.0581381 & 35.0658395 & -6.9644712 & 0.4740158 \\
\hline & 3 & -444.6620029 & 913.3400658 & -347.7358123 & 51.9244201 & -2.7150328 \\
\hline \multirow{3}{*}{$\mathrm{H}_{\beta}$} & 1 & -0.4120752 & 0.9250669 & -0.3741119 & 0.0622541 & -0.0036591 \\
\hline & 2 & 26.4414835 & -74.8894421 & 48.1881455 & -11.2855830 & 0.8626001 \\
\hline & 3 & -805.0295817 & 2280.7328848 & -1494.4764420 & 349.3195439 & -26.6678020 \\
\hline \multirow{3}{*}{ Fe5015 } & 1 & -0.0313311 & -0.0500534 & -0.0035580 & -0.0037280 & 0.0007775 \\
\hline & 2 & 61.9785871 & -107.1406403 & 40.6549692 & -4.5784440 & 0.0744308 \\
\hline & 3 & -1490.9225648 & 2286.4644788 & -734.9273433 & 46.6787692 & 3.5030950 \\
\hline \multirow{3}{*}{$\mathrm{Mg}_{1}$} & 1 & -0.0011669 & 0.0020035 & -0.0025450 & 0.0006800 & -0.0000553 \\
\hline & 2 & 1.0977834 & -1.6954874 & 0.4725610 & -0.0265407 & -0.0021062 \\
\hline & 3 & -23.8411177 & 30.5518215 & -4.5747311 & -1.0811535 & 0.1935160 \\
\hline \multirow{3}{*}{$\mathrm{Mg}_{2}$} & 1 & -0.0036972 & 0.0016391 & -0.0002221 & -0.0002245 & 0.0000340 \\
\hline & 2 & 2.6546194 & -4.0910074 & 1.1294327 & -0.0429887 & -0.0081361 \\
\hline & 3 & -63.2072524 & 93.1999501 & -25.0701341 & 0.5006265 & 0.2481080 \\
\hline \multirow{3}{*}{$\mathrm{Mg}_{\mathrm{b}}$} & 1 & -0.1159519 & 0.0564072 & 0.0367218 & -0.0207147 & 0.0023015 \\
\hline & 2 & 36.6456419 & -47.5077586 & 8.8070472 & 1.2388375 & -0.2750762 \\
\hline & 3 & -857.5383388 & 1080.0808279 & -207.5498638 & -26.8404813 & 6.2521890 \\
\hline \multirow{3}{*}{$\mathrm{Fe} 5270$} & 1 & 0.1443967 & -0.2658498 & 0.0907173 & -0.0111382 & 0.0003331 \\
\hline & 2 & -8.5450001 & 12.6759249 & -7.5470939 & 1.5641500 & -0.0991560 \\
\hline & 3 & 306.8420600 & -637.6523032 & 403.3286320 & -89.3943857 & 6.3626512 \\
\hline
\end{tabular}


Table 1 -continued.

\begin{tabular}{|c|c|c|c|c|c|c|}
\hline Index & $j$ & $\mathrm{C}_{1 \mathrm{j}}$ & $\mathrm{C}_{2 \mathrm{j}}$ & $\mathrm{C}_{3 \mathrm{j}}$ & $\mathrm{C}_{4 \mathrm{j}}$ & $\mathrm{C}_{5 \mathrm{j}}$ \\
\hline \multirow{3}{*}{ Fe5335 } & 1 & 0.1121199 & -0.2143513 & 0.0776458 & -0.0095392 & 0.0002385 \\
\hline & 2 & -6.1989089 & 11.7530508 & -8.2144476 & 1.6722406 & -0.0990246 \\
\hline & 3 & 144.7440581 & -352.4412737 & 218.9898664 & -44.2594619 & 2.7694391 \\
\hline \multirow{3}{*}{ Fe5406 } & 1 & -0.0167597 & -0.0094937 & -0.0069105 & 0.0022746 & -0.0001900 \\
\hline & 2 & 18.1518764 & -28.2532163 & 9.0539150 & -0.8243859 & -0.0014094 \\
\hline & 3 & -423.9701730 & 596.6530611 & -158.4751506 & 4.4556600 & 1.3344343 \\
\hline \multirow{3}{*}{ Fe5709 } & 1 & 0.0643501 & -0.1189715 & 0.0352640 & -0.0029869 & -0.0000373 \\
\hline & 2 & -6.8927462 & 9.4915829 & -2.5896330 & 0.0686243 & 0.0256903 \\
\hline & 3 & 206.1312747 & -349.9572257 & 151.0604011 & -22.2154935 & 0.9194484 \\
\hline \multirow{3}{*}{ Fe5782 } & 1 & -0.0193549 & 0.0201941 & -0.0227794 & 0.0068166 & -0.0006059 \\
\hline & 2 & 5.8734339 & -8.3105593 & 3.3800221 & -0.6333537 & 0.0435585 \\
\hline & 3 & -145.6309054 & 234.0700497 & -130.6085667 & 28.1896236 & -2.0267932 \\
\hline \multirow{3}{*}{$\mathrm{Na}_{\mathrm{D}}$} & 1 & -0.1041283 & 0.0782159 & -0.0131910 & -0.0028878 & 0.0005590 \\
\hline & 2 & 35.8174765 & -52.1821670 & 16.3021886 & -1.2441912 & -0.0404260 \\
\hline & 3 & -928.3347064 & 1387.5693179 & -484.7687597 & 49.3839423 & -0.3018862 \\
\hline \multirow{3}{*}{$\mathrm{TiO}_{1}$} & 1 & -0.0046972 & -0.0031675 & 0.0086166 & -0.0031273 & 0.0003031 \\
\hline & 2 & 2.5376502 & -3.1526728 & 0.3308384 & 0.2054016 & -0.0316500 \\
\hline & 3 & -70.3829758 & 95.4015845 & -17.9727317 & -3.0711691 & 0.6438944 \\
\hline \multirow{3}{*}{$\mathrm{TiO}_{2}$} & 1 & -0.0089825 & -0.0020920 & 0.0096106 & -0.0036755 & 0.0003656 \\
\hline & 2 & 3.9165850 & -4.9045789 & 0.8074595 & 0.2009710 & -0.0375203 \\
\hline & 3 & -108.7948038 & 146.4687943 & -32.9218404 & -2.5469339 & 0.7759394 \\
\hline \multirow{3}{*}{$\mathrm{H} \delta_{\mathrm{A}}$} & 1 & -0.3400174 & 0.7802154 & -0.3437893 & 0.0824056 & -0.0066930 \\
\hline & 2 & 36.1459627 & -178.0526116 & 166.3449941 & -45.4458738 & 3.7573350 \\
\hline & 3 & -1381.3144000 & 5283.2867289 & -4334.0624242 & 1125.3443000 & -90.9465139 \\
\hline \multirow{3}{*}{$\mathrm{H} \gamma_{\mathrm{A}}$} & 1 & -0.7586478 & 1.6470466 & -0.6800223 & 0.1306246 & -0.0086650 \\
\hline & 2 & 58.8625436 & -210.0639058 & 174.8989489 & -45.3356756 & 3.6089852 \\
\hline & 3 & -2043.5700337 & 6433.0522877 & -4883.0699797 & 1207.4959655 & -93.9809592 \\
\hline \multirow{3}{*}{$\mathrm{H} \delta_{\mathrm{F}}$} & 1 & -0.3060721 & 0.7125074 & -0.3205038 & 0.0683519 & -0.0050475 \\
\hline & 2 & 30.6677991 & -121.8747783 & 101.4148311 & -26.5930774 & 2.1537733 \\
\hline & 3 & -1053.7758076 & 3611.9972771 & -2723.9526032 & 682.2344274 & -54.0667213 \\
\hline \multirow{3}{*}{$\mathrm{H} \gamma_{\mathrm{F}}$} & 1 & -0.4440136 & 0.9835898 & -0.4158944 & 0.0801404 & -0.0053903 \\
\hline & 2 & 35.2952498 & -120.5372324 & 95.1523223 & -24.3827679 & 1.9426707 \\
\hline & 3 & -1145.3060328 & 3586.2350776 & -2658.3958905 & 655.6380015 & -51.2487311 \\
\hline
\end{tabular}


Table 2 Similar to Table 1, but for stellar populations older than 4 Gyr (Age $\geq$ 4 Gyr).

\begin{tabular}{|c|c|c|c|c|c|c|}
\hline Index & $j$ & $\mathrm{C}_{1 \mathrm{j}}$ & $\mathrm{C}_{2 \mathrm{j}}$ & $\mathrm{C}_{3 \mathrm{j}}$ & $\mathrm{C}_{4 \mathrm{j}}$ & $\mathrm{C}_{5 \mathrm{j}}$ \\
\hline \multirow{3}{*}{$\mathrm{CN}_{1}$} & 1 & 0.0040188 & -0.0087338 & 0.0012365 & -0.0000628 & 0.0000011 \\
\hline & 2 & 0.0699149 & -0.5550663 & 0.1605598 & -0.0163788 & 0.0005280 \\
\hline & 3 & -4.2835098 & 11.3189732 & -2.8137761 & 0.2856955 & -0.0093980 \\
\hline \multirow{3}{*}{$\mathrm{CN}_{2}$} & 1 & 0.0031607 & -0.0068581 & 0.0009171 & -0.0000386 & 0.0000004 \\
\hline & 2 & 0.1358253 & -0.6142418 & 0.1616565 & -0.0161493 & 0.0005224 \\
\hline & 3 & -5.8469187 & 12.5102930 & -2.9058639 & 0.2889257 & -0.0095349 \\
\hline \multirow{3}{*}{$\mathrm{Ca} 4227$} & 1 & 0.0024019 & -0.0096646 & 0.0028396 & -0.0003487 & 0.0000134 \\
\hline & 2 & 0.1289259 & -0.3224843 & -0.1484763 & 0.0361270 & -0.0016691 \\
\hline & 3 & 30.5865753 & -70.1598679 & 19.3765757 & -2.1609623 & 0.0788180 \\
\hline \multirow{3}{*}{ G4300 } & 1 & 0.1365771 & -0.4119753 & 0.0710301 & -0.0055584 & 0.0001697 \\
\hline & 2 & -3.6369924 & 2.4500207 & 0.5456707 & -0.0466573 & -0.0003761 \\
\hline & 3 & 28.2611775 & -85.3355224 & 18.7904056 & -2.1578069 & 0.1062849 \\
\hline \multirow{3}{*}{$\mathrm{Fe} 4383$} & 1 & 0.0695695 & -0.1937149 & 0.0295201 & -0.0017795 & 0.0000416 \\
\hline & 2 & 1.5938861 & -9.3601744 & 2.3994053 & -0.2263281 & 0.0068871 \\
\hline & 3 & -79.2726970 & 189.8780408 & -38.5276774 & 3.5614204 & -0.1108842 \\
\hline \multirow{3}{*}{ Ca4455 } & 1 & 0.0113051 & -0.0260901 & 0.0031068 & -0.0001249 & 0.0000015 \\
\hline & 2 & 0.1825335 & -2.1257283 & 0.6162648 & -0.0626674 & 0.0020383 \\
\hline & 3 & -18.3660159 & 52.8898164 & -13.1167084 & 1.3350018 & -0.0447127 \\
\hline \multirow{3}{*}{$\mathrm{Fe} 4531$} & 1 & 0.0241263 & -0.1055130 & 0.0195680 & -0.0014870 & 0.0000418 \\
\hline & 2 & -0.8767742 & -0.8527878 & 0.3649493 & -0.0354193 & 0.0009578 \\
\hline & 3 & 25.2479921 & -36.0000062 & 6.6317767 & -0.5259928 & 0.0185966 \\
\hline \multirow{3}{*}{$\mathrm{Fe} 4668$} & 1 & 0.0179749 & -0.0676067 & 0.0083280 & -0.0002240 & -0.0000019 \\
\hline & 2 & 0.2262582 & -4.4121200 & 1.5353953 & -0.1944241 & 0.0071387 \\
\hline & 3 & -79.5135908 & 227.1468134 & -55.0061793 & 6.0957633 & -0.2199873 \\
\hline \multirow{3}{*}{$\mathrm{H}_{\beta}$} & 1 & -0.0625565 & 0.2708823 & -0.0566269 & 0.0045862 & -0.0001317 \\
\hline & 2 & 2.8700663 & -5.7104589 & 0.9274182 & -0.0705995 & 0.0022889 \\
\hline & 3 & -40.1033590 & 83.2554372 & -18.0609224 & 1.5794001 & -0.0541752 \\
\hline \multirow{3}{*}{ Fe5015 } & 1 & 0.0326274 & -0.1721448 & 0.0331789 & -0.0025354 & 0.0000699 \\
\hline & 2 & -1.1476034 & -1.5307235 & 0.5946861 & -0.0674897 & 0.0022440 \\
\hline & 3 & 7.2743924 & 22.9005565 & -3.9738686 & 0.4451390 & -0.0154651 \\
\hline \multirow{3}{*}{$\mathrm{Mg}_{1}$} & 1 & 0.0002583 & -0.0028680 & 0.0005454 & -0.0000406 & 0.0000011 \\
\hline & 2 & 0.0303274 & -0.1102308 & 0.0115624 & -0.0003957 & -0.0000009 \\
\hline & 3 & -0.0290541 & 0.0201172 & 0.2573529 & -0.0302840 & 0.0011283 \\
\hline \multirow{3}{*}{$\mathrm{Mg}_{2}$} & 1 & 0.0003494 & -0.0047501 & 0.0010250 & -0.0000857 & 0.0000026 \\
\hline & 2 & 0.0392009 & -0.1979893 & 0.0208337 & -0.0000621 & -0.0000425 \\
\hline & 3 & 0.4638829 & -0.5123346 & 0.6866533 & -0.0971528 & 0.0039890 \\
\hline \multirow{3}{*}{$\mathrm{Mg}_{\mathrm{b}}$} & 1 & -0.0037660 & -0.0157034 & 0.0047387 & -0.0004621 & 0.0000160 \\
\hline & 2 & 0.9698109 & -4.0019894 & 0.5949929 & -0.0254183 & 0.0000838 \\
\hline & 3 & -10.4139848 & 42.6783675 & -1.0978610 & -0.5308182 & 0.0325709 \\
\hline \multirow{3}{*}{ Fe5270 } & 1 & 0.0135064 & -0.0676152 & 0.0131365 & -0.0009717 & 0.0000261 \\
\hline & 2 & -0.3787006 & -1.1509906 & 0.2995794 & -0.0297612 & 0.0009629 \\
\hline & 3 & 2.9257542 & 20.0839626 & -2.9644946 & 0.2873734 & -0.0103891 \\
\hline
\end{tabular}


Table 2 -continued.

\begin{tabular}{|c|c|c|c|c|c|c|}
\hline Index & $j$ & $\mathrm{C}_{1 \mathrm{j}}$ & $\mathrm{C}_{2 \mathrm{j}}$ & $\mathrm{C}_{3 \mathrm{j}}$ & $\mathrm{C}_{4 \mathrm{j}}$ & $\mathrm{C}_{5 \mathrm{j}}$ \\
\hline \multirow{3}{*}{ Fe5335 } & 1 & 0.0100735 & -0.0513031 & 0.0112253 & -0.0009878 & 0.0000303 \\
\hline & 2 & -0.3764122 & -1.1131799 & -0.1092792 & 0.0392520 & -0.0018016 \\
\hline & 3 & 46.0187216 & -72.8319926 & 27.5059454 & -3.2158884 & 0.1133870 \\
\hline \multirow{3}{*}{ Fe5406 } & 1 & 0.0061912 & -0.0466338 & 0.0093712 & -0.0007327 & 0.0000206 \\
\hline & 2 & 0.0934871 & -1.3125122 & 0.1939528 & -0.0108703 & 0.0001877 \\
\hline & 3 & 1.0004186 & 10.2495446 & 1.2408277 & -0.2790750 & 0.0120963 \\
\hline \multirow{3}{*}{ Fe5709 } & 1 & 0.0084714 & -0.0287635 & 0.0050787 & -0.0003500 & 0.0000089 \\
\hline & 2 & -0.4407574 & 0.0360397 & 0.0939359 & -0.0153071 & 0.0006047 \\
\hline & 3 & -0.8112771 & 19.1722212 & -4.5075237 & 0.4973342 & -0.0184540 \\
\hline \multirow{3}{*}{ Fe5782 } & 1 & -0.0002944 & -0.0149229 & 0.0032162 & -0.0003063 & 0.0000099 \\
\hline & 2 & 0.2419052 & -0.2990725 & -0.1171528 & 0.0246488 & -0.0010394 \\
\hline & 3 & 20.8520931 & -52.8185426 & 16.2838692 & -1.7500469 & 0.0595642 \\
\hline \multirow{3}{*}{$\mathrm{Na}_{\mathrm{D}}$} & 1 & -0.0022021 & -0.0380672 & 0.0095990 & -0.0008472 & 0.0000260 \\
\hline & 2 & 0.3464177 & -0.9169326 & -0.0149337 & 0.0195706 & -0.0010391 \\
\hline & 3 & 14.6152128 & -38.0493377 & 12.0162242 & -1.3802231 & 0.0519050 \\
\hline \multirow{3}{*}{$\mathrm{TiO}_{1}$} & 1 & -0.0004048 & -0.0010965 & 0.0002912 & -0.0000264 & 0.0000008 \\
\hline & 2 & 0.0098709 & -0.0060943 & -0.0015886 & 0.0001050 & -0.0000058 \\
\hline & 3 & 0.4022334 & -0.0305906 & -0.0854966 & 0.0157629 & -0.0004537 \\
\hline \multirow{3}{*}{$\mathrm{TiO}_{2}$} & 1 & -0.0006406 & -0.0026937 & 0.0006705 & -0.0000589 & 0.0000018 \\
\hline & 2 & 0.0411593 & 0.0211796 & -0.0159178 & 0.0015739 & -0.0000518 \\
\hline & 3 & -0.4009774 & -0.3431377 & 0.1546906 & -0.0093757 & 0.0003560 \\
\hline \multirow{3}{*}{$\mathrm{H} \delta_{\mathrm{A}}$} & 1 & -0.1822723 & 0.4528323 & -0.0619613 & 0.0036523 & -0.0000907 \\
\hline & 2 & -9.3242935 & 26.8376168 & -7.6220614 & 0.6779245 & -0.0184436 \\
\hline & 3 & 273.5136304 & -384.4491796 & 87.5549531 & -6.8624029 & 0.1483407 \\
\hline \multirow{3}{*}{$\mathrm{H} \gamma_{\mathrm{A}}$} & 1 & -0.2347224 & 0.6562770 & -0.0985331 & 0.0067082 & -0.0001900 \\
\hline & 2 & -8.3702686 & 24.2585985 & -7.8246936 & 0.7094328 & -0.0186995 \\
\hline & 3 & 392.7692657 & -531.6987235 & 129.8789712 & -11.2146737 & 0.2783352 \\
\hline \multirow{3}{*}{$\mathrm{H} \delta_{\mathrm{F}}$} & 1 & -0.1106862 & 0.2930026 & -0.0443820 & 0.0029922 & -0.0000815 \\
\hline & 2 & -3.5060331 & 10.7215430 & -3.5595302 & 0.3279227 & -0.0089577 \\
\hline & 3 & 145.3867861 & -162.5243753 & 44.0186086 & -3.7498376 & 0.0877458 \\
\hline \multirow{3}{*}{$\mathrm{H} \gamma_{\mathrm{F}}$} & 1 & -0.1284349 & 0.3643152 & -0.0583081 & 0.0041664 & -0.0001196 \\
\hline & 2 & -1.3760493 & 7.0593899 & -2.8690465 & 0.2678504 & -0.0067945 \\
\hline & 3 & 129.2354201 & -186.3215690 & 49.9193880 & -4.3279933 & 0.0997893 \\
\hline
\end{tabular}


Table 3 Coefficients for equation (2). UBVRIJHKLMN magnitudes are on Johnson system, and ugriz magnitudes are on SDSS-ugriz system.

\begin{tabular}{|c|c|c|c|c|c|c|c|c|}
\hline IMF & \multicolumn{3}{|c|}{ Salpeter } & & \multicolumn{4}{|c|}{ Chabrier } \\
\hline \multicolumn{9}{|c|}{ Age $<4.2 \mathrm{Gyr}$} \\
\hline Colour & $\mathrm{C}_{1}$ & $\mathrm{C}_{2}$ & $\mathrm{C}_{3}$ & $\mathrm{C}_{4}$ & $\mathrm{C}_{1}$ & $\mathrm{C}_{2}$ & $\mathrm{C}_{3}$ & $\mathrm{C}_{4}$ \\
\hline$(\mathrm{B}-\mathrm{V})$ & -0.014222 & -0.032764 & 0.009111 & -0.000722 & -0.020059 & -0.021895 & 0.005569 & -0.000449 \\
\hline$(\mathrm{V}-\mathrm{K})$ & -0.080134 & -0.093961 & 0.038424 & -0.004241 & -0.086556 & -0.062010 & 0.021278 & -0.001931 \\
\hline$(\mathrm{I}-\mathrm{H})$ & -0.047703 & -0.049909 & 0.021701 & -0.002461 & -0.049641 & -0.033034 & 0.011729 & -0.001032 \\
\hline$(\mathrm{R}-\mathrm{K})$ & -0.043288 & -0.037439 & 0.016264 & -0.001812 & -0.042418 & -0.025391 & 0.008674 & -0.000701 \\
\hline$(\mathrm{B}-\mathrm{K})$ & -0.094328 & -0.126572 & 0.047424 & -0.004947 & -0.106087 & -0.084725 & 0.027181 & -0.002419 \\
\hline$(\mathrm{I}-\mathrm{K})$ & -0.054306 & -0.055956 & 0.024500 & -0.002787 & -0.055634 & -0.036844 & 0.012924 & -0.001109 \\
\hline$(\mathrm{u}-\mathrm{r})$ & -0.056601 & -0.042940 & 0.012347 & -0.001002 & -0.063465 & -0.023075 & 0.005139 & -0.000410 \\
\hline$(\mathrm{r}-\mathrm{K})$ & -0.072748 & -0.083108 & 0.035437 & -0.004014 & -0.077569 & -0.054339 & 0.019333 & -0.001782 \\
\hline$(\mathrm{u}-\mathrm{R})$ & -0.059219 & -0.047988 & 0.014264 & -0.001213 & -0.066913 & -0.026108 & 0.006208 & -0.000525 \\
\hline$(\mathrm{u}-\mathrm{K})$ & -0.129146 & -0.126222 & 0.047806 & -0.005016 & -0.140906 & -0.077425 & 0.024514 & -0.002201 \\
\hline$(\mathrm{z}-\mathrm{K})$ & -0.043288 & -0.037439 & 0.016264 & -0.001812 & -0.042418 & -0.025391 & 0.008674 & -0.000701 \\
\hline$(\mathrm{g}-\mathrm{J})$ & -0.065198 & -0.092019 & 0.035014 & -0.003704 & -0.074778 & -0.061406 & 0.020278 & -0.001863 \\
\hline \multicolumn{9}{|c|}{ Age $\geq 4.2 \mathrm{Gyr}$} \\
\hline Colour & $\mathrm{C}_{1}$ & $\mathrm{C}_{2}$ & $\mathrm{C}_{3}$ & $\mathrm{C}_{4}$ & $\mathrm{C}_{1}$ & $\mathrm{C}_{2}$ & $\mathrm{C}_{3}$ & $\mathrm{C}_{4}$ \\
\hline$(\mathrm{B}-\mathrm{V})$ & -0.069795 & 0.008858 & -0.000767 & 0.000025 & -0.062030 & 0.005088 & -0.000384 & 0.000014 \\
\hline$(\mathrm{V}-\mathrm{K})$ & -0.157537 & 0.014833 & -0.001013 & 0.000025 & -0.191178 & 0.028283 & -0.002893 & 0.000103 \\
\hline$(\mathrm{I}-\mathrm{H})$ & -0.087919 & 0.010592 & -0.000813 & 0.000020 & -0.113589 & 0.020964 & -0.002196 & 0.000076 \\
\hline$(\mathrm{R}-\mathrm{K})$ & -0.078362 & 0.010635 & -0.000850 & 0.000022 & -0.099112 & 0.019317 & -0.002018 & 0.000069 \\
\hline$(\mathrm{B}-\mathrm{K})$ & -0.222114 & 0.020910 & -0.001424 & 0.000037 & -0.245483 & 0.029974 & -0.002870 & 0.000102 \\
\hline$(\mathrm{I}-\mathrm{K})$ & -0.100169 & 0.012567 & -0.000978 & 0.000024 & -0.130611 & 0.024976 & -0.002622 & 0.000090 \\
\hline$(\mathrm{u}-\mathrm{r})$ & -0.119987 & 0.009885 & -0.001017 & 0.000042 & -0.098610 & 0.000563 & -0.000163 & 0.000019 \\
\hline$(\mathrm{r}-\mathrm{K})$ & -0.135641 & 0.013834 & -0.001022 & 0.000026 & -0.169588 & 0.027336 & -0.002877 & 0.000102 \\
\hline$(\mathrm{u}-\mathrm{R})$ & -0.126930 & 0.010203 & -0.001026 & 0.000042 & -0.106134 & 0.001228 & -0.000236 & 0.000022 \\
\hline$(\mathrm{u}-\mathrm{K})$ & -0.255286 & 0.021748 & -0.001696 & 0.000052 & -0.264095 & 0.024796 & -0.002561 & 0.000101 \\
\hline$(\mathrm{z}-\mathrm{K})$ & -0.078362 & 0.010635 & -0.000850 & 0.000022 & -0.099112 & 0.019317 & -0.002018 & 0.000069 \\
\hline$(\mathrm{g}-\mathrm{J})$ & -0.154783 & 0.014291 & -0.000930 & 0.000024 & -0.171114 & 0.020427 & -0.001897 & 0.000067 \\
\hline
\end{tabular}


Table A.1 Coefficients for equation (1). The coefficients are obtained via stellar populations with Chabrier IMF and can be used for populations younger than $3.5 \mathrm{Gyr}$ (Age $<3.5$ Gyr).

\begin{tabular}{|c|c|c|c|c|c|c|}
\hline Index & $j$ & $\mathrm{C}_{1 \mathrm{j}}$ & $\mathrm{C}_{2 \mathrm{j}}$ & $\mathrm{C}_{3 \mathrm{j}}$ & $\mathrm{C}_{4 \mathrm{j}}$ & $\mathrm{C}_{5 \mathrm{j}}$ \\
\hline \multirow{3}{*}{$\mathrm{CN}_{1}$} & 1 & 0.0022919 & 0.0011051 & -0.0063997 & 0.0015981 & -0.0001080 \\
\hline & 2 & 0.6329579 & -0.9595824 & 0.2427491 & -0.0011151 & -0.0025634 \\
\hline & 3 & -4.5940253 & 7.9284009 & -3.9250539 & 0.6480545 & -0.0256989 \\
\hline \multirow{3}{*}{$\mathrm{CN}_{2}$} & 1 & 0.0012774 & 0.0032128 & -0.0069053 & 0.0016921 & -0.0001156 \\
\hline & 2 & 0.6334017 & -1.0656913 & 0.3400438 & -0.0309074 & -0.0000476 \\
\hline & 3 & -4.4675288 & 9.5105511 & -5.7521116 & 1.2233685 & -0.0747440 \\
\hline \multirow{3}{*}{$\mathrm{Ca} 4227$} & 1 & 0.0795038 & -0.1571070 & 0.0740613 & -0.0128663 & 0.0007442 \\
\hline & 2 & -11.9558663 & 20.8851588 & -9.0611781 & 1.3421325 & -0.0622930 \\
\hline & 3 & 342.8235941 & -529.6545151 & 161.4994646 & -12.9148165 & -0.1134096 \\
\hline \multirow{3}{*}{ G4300 } & 1 & 0.7660019 & -1.5947618 & 0.6186025 & -0.0971560 & 0.0052551 \\
\hline & 2 & -87.0440009 & 155.4728799 & -65.2201097 & 9.5672170 & -0.4355927 \\
\hline & 3 & 2358.1318663 & -4145.5253913 & 1656.4267226 & -221.2634252 & 8.6149062 \\
\hline \multirow{3}{*}{$\mathrm{Fe} 4383$} & 1 & 0.6089788 & -0.9690479 & 0.3009590 & -0.0329757 & 0.0009352 \\
\hline & 2 & -72.6995453 & 111.7196061 & -45.0101690 & 6.0333694 & -0.2331824 \\
\hline & 3 & 2100.1581312 & -3361.5113576 & 1335.7159550 & -174.8630448 & 6.5371492 \\
\hline \multirow{3}{*}{$\mathrm{Ca} 4455$} & 1 & 0.0681495 & -0.1369378 & 0.0592712 & -0.0105410 & 0.0006446 \\
\hline & 2 & -7.3288591 & 13.2396365 & -7.0194851 & 1.2883776 & -0.0767611 \\
\hline & 3 & 258.9163092 & -510.1718798 & 260.9018384 & -46.2914894 & 2.6884347 \\
\hline \multirow{3}{*}{$\mathrm{Fe} 4531$} & 1 & 0.2564424 & -0.5328445 & 0.2229634 & -0.0364862 & 0.0020371 \\
\hline & 2 & -26.0845865 & 42.1057901 & -17.3504899 & 2.4029055 & -0.0987445 \\
\hline & 3 & 851.0453910 & -1492.6215470 & 616.0271336 & -86.7449029 & 3.7433351 \\
\hline \multirow{3}{*}{ Fe4668 } & 1 & 0.1275239 & -0.2791766 & 0.1024538 & -0.0161675 & 0.0009372 \\
\hline & 2 & -5.2389930 & 7.6979073 & -2.3867283 & 0.1846930 & -0.0012464 \\
\hline & 3 & 169.0223930 & -536.0764913 & 339.6448905 & -65.7521400 & 4.1760430 \\
\hline \multirow{3}{*}{$\mathrm{H}_{\beta}$} & 1 & -0.4293708 & 0.8954661 & -0.3155113 & 0.0432326 & -0.0020233 \\
\hline & 2 & 30.1096288 & -49.0583758 & 16.8580314 & -1.8838154 & 0.0479103 \\
\hline & 3 & -767.8393985 & 1229.9316335 & -430.0651511 & 45.5750506 & -0.8795314 \\
\hline \multirow{3}{*}{ Fe5015 } & 1 & 0.2395560 & -0.3782478 & 0.1010824 & -0.0116568 & 0.0005056 \\
\hline & 2 & -3.3348359 & -34.6665677 & 26.0424965 & -5.8877139 & 0.4132806 \\
\hline & 3 & 413.3734390 & 45.5824955 & -217.1527754 & 66.7394428 & -5.4107732 \\
\hline \multirow{3}{*}{$\mathrm{Mg}_{1}$} & 1 & 0.0057639 & -0.0122970 & 0.0048864 & -0.0007787 & 0.0000435 \\
\hline & 2 & -0.3355850 & 0.6283524 & -0.2850889 & 0.0319693 & -0.0005680 \\
\hline & 3 & 13.7239823 & -27.6095038 & 10.7666288 & -1.0488923 & 0.0060013 \\
\hline \multirow{3}{*}{$\mathrm{Mg}_{2}$} & 1 & 0.0123326 & -0.0261803 & 0.0111134 & -0.0018290 & 0.0001038 \\
\hline & 2 & -0.7115473 & 1.0153443 & -0.3612812 & 0.0205355 & 0.0016030 \\
\hline & 3 & 27.2273352 & -44.1981147 & 13.4974485 & -0.5084821 & -0.0847616 \\
\hline \multirow{3}{*}{$\mathrm{Mg}_{\mathrm{b}}$} & 1 & 0.1259541 & -0.2256908 & 0.0880777 & -0.0124308 & 0.0005828 \\
\hline & 2 & -8.2670312 & 5.7969056 & 0.6635323 & -0.9033180 & 0.1008251 \\
\hline & 3 & 311.7946049 & -348.4131106 & 36.5555001 & 20.8760850 & -2.8507846 \\
\hline \multirow{3}{*}{ Fe5270 } & 1 & 0.1526957 & -0.3633561 & 0.1689542 & -0.0304730 & 0.0018686 \\
\hline & 2 & -16.8516642 & 33.9221551 & -18.3927017 & 3.5096217 & -0.2189850 \\
\hline & 3 & 587.4969012 & -1243.1886864 & 678.5522252 & -131.0104877 & 8.3026556 \\
\hline
\end{tabular}


Table A.1 -continued.

\begin{tabular}{|c|c|c|c|c|c|c|}
\hline Index & $j$ & $\mathrm{C}_{1 \mathrm{j}}$ & $\mathrm{C}_{2 \mathrm{j}}$ & $\mathrm{C}_{3 \mathrm{j}}$ & $\mathrm{C}_{4 \mathrm{j}}$ & $\mathrm{C}_{5 \mathrm{j}}$ \\
\hline Fe5335 & $\begin{array}{l}1 \\
2 \\
3 \\
\end{array}$ & $\begin{array}{r}0.2076133 \\
-32.4556974 \\
935.2591701 \\
\end{array}$ & $\begin{array}{r}-0.4791791 \\
65.1973802 \\
-1881.6098576 \\
\end{array}$ & $\begin{array}{r}0.2222679 \\
-31.8891247 \\
872.5249261 \\
\end{array}$ & $\begin{array}{r}-0.0377229 \\
5.4057781 \\
-142.2695305 \\
\end{array}$ & $\begin{array}{r}0.0021281 \\
-0.2980594 \\
7.5855211 \\
\end{array}$ \\
\hline Fe5406 & $\begin{array}{l}1 \\
2 \\
3 \\
\end{array}$ & $\begin{array}{r}0.0953714 \\
-3.9285567 \\
174.8445638 \\
\end{array}$ & $\begin{array}{r}-0.2161430 \\
6.4393293 \\
-345.4890330 \\
\end{array}$ & $\begin{array}{r}0.0970755 \\
-3.6907976 \\
172.7005100 \\
\end{array}$ & $\begin{array}{r}-0.0171741 \\
0.6355834 \\
-28.2035654 \\
\end{array}$ & $\begin{array}{r}0.0010419 \\
-0.0343946 \\
1.4789489 \\
\end{array}$ \\
\hline Fe5709 & $\begin{array}{l}1 \\
2 \\
3 \\
\end{array}$ & $\begin{array}{r}0.0665533 \\
-8.4184254 \\
229.4380018 \\
\end{array}$ & $\begin{array}{r}-0.1333817 \\
11.6155577 \\
-346.7249858 \\
\end{array}$ & $\begin{array}{r}0.0460766 \\
-2.8870447 \\
109.9625205 \\
\end{array}$ & $\begin{array}{r}-0.0056987 \\
0.0168183 \\
-7.5674339 \\
\end{array}$ & $\begin{array}{r}0.0002133 \\
0.0277514 \\
-0.2639790 \\
\end{array}$ \\
\hline Fe5782 & $\begin{array}{l}1 \\
2 \\
3 \\
\end{array}$ & $\begin{array}{r}0.0724796 \\
-12.3652716 \\
379.6596477 \\
\end{array}$ & $\begin{array}{r}-0.1553226 \\
23.1281644 \\
-665.9564600 \\
\end{array}$ & $\begin{array}{r}0.0682675 \\
-10.7411540 \\
268.9228630 \\
\end{array}$ & $\begin{array}{r}-0.0111777 \\
1.7511767 \\
-38.4448161 \\
\end{array}$ & $\begin{array}{r}0.0006131 \\
-0.0935373 \\
1.7703055 \\
\end{array}$ \\
\hline $\mathrm{Na}_{\mathrm{D}}$ & $\begin{array}{l}1 \\
2 \\
3 \\
\end{array}$ & $\begin{array}{r}0.0714688 \\
-1.5605878 \\
148.3896060 \\
\end{array}$ & $\begin{array}{r}-0.1651977 \\
-4.9385375 \\
-24.0899505 \\
\end{array}$ & $\begin{array}{r}0.0664805 \\
5.3394247 \\
-130.0698652 \\
\end{array}$ & $\begin{array}{l}-0.0103341 \\
-1.5674185 \\
49.4638805 \\
\end{array}$ & $\begin{array}{r}0.0005679 \\
0.1304109 \\
-4.4865514 \\
\end{array}$ \\
\hline $\mathrm{TiO}_{1}$ & $\begin{array}{l}1 \\
2 \\
3\end{array}$ & $\begin{array}{r}-0.0115706 \\
2.0705973 \\
-44.5370053\end{array}$ & $\begin{array}{r}0.0112891 \\
-2.9503555 \\
67.5680594\end{array}$ & $\begin{array}{r}-0.0010864 \\
0.8324854 \\
-20.7546877\end{array}$ & $\begin{array}{r}-0.0006067 \\
-0.0401368 \\
1.4636658\end{array}$ & $\begin{array}{r}0.0000840 \\
-0.0040348 \\
0.0462995\end{array}$ \\
\hline $\mathrm{TiO}_{2}$ & 3 & $\begin{array}{r}-0.0125496 \\
2.4479466 \\
-49.2619296 \\
\end{array}$ & $\begin{array}{r}0.0097243 \\
-3.6429429 \\
79.6645168 \\
\end{array}$ & $\begin{array}{r}-0.0001968 \\
1.1610628 \\
-27.3299096 \\
\end{array}$ & $\begin{array}{r}-0.0008541 \\
-0.0929928 \\
2.6274999 \\
\end{array}$ & $\begin{array}{r}0.0001063 \\
-0.0014161 \\
-0.0183345 \\
\end{array}$ \\
\hline $\mathrm{H} \delta_{\mathrm{A}}$ & 3 & $\begin{array}{r}-0.1701616 \\
-22.7201592 \\
35.0832106 \\
\end{array}$ & $\begin{array}{r}0.1253346 \\
34.6318625 \\
-330.3012402 \\
\end{array}$ & $\begin{array}{r}0.2496936 \\
-16.9398410 \\
581.7572135 \\
\end{array}$ & $\begin{array}{r}-0.0688490 \\
2.8138566 \\
-167.8682957 \\
\end{array}$ & $\begin{array}{r}0.0048300 \\
-0.1414102 \\
12.8850332 \\
\end{array}$ \\
\hline $\mathrm{H} \gamma_{\mathrm{A}}$ & 3 & $\begin{array}{r}-1.5856131 \\
180.2526812 \\
-5010.4626342\end{array}$ & $\begin{array}{r}2.7085039 \\
-282.6477080 \\
7614.1515475\end{array}$ & $\begin{array}{r}-0.9113125 \\
118.3329534 \\
-2881.3416065\end{array}$ & $\begin{array}{r}0.1227389 \\
-17.2376118 \\
351.0737254 \\
\end{array}$ & $\begin{array}{r}-0.0055250 \\
0.7715612 \\
-11.0363129\end{array}$ \\
\hline $\mathrm{H} \delta_{\mathrm{F}}$ & 3 & $\begin{array}{r}-0.1251870 \\
-17.3098016 \\
137.3583135\end{array}$ & $\begin{array}{r}0.1470139 \\
30.7053467 \\
-435.8552796\end{array}$ & $\begin{array}{r}0.1086295 \\
-17.0765539 \\
470.4948769\end{array}$ & $\begin{array}{r}-0.0343830 \\
3.2519508 \\
-124.2942197\end{array}$ & $\begin{array}{r}0.0025515 \\
-0.1978084 \\
9.3212486\end{array}$ \\
\hline $\mathrm{H} \gamma_{\mathrm{F}}$ & 3 & $\begin{array}{r}-0.6254725 \\
53.4441919 \\
-1532.4775410\end{array}$ & $\begin{array}{r}1.1213832 \\
-86.7513473 \\
2361.4227142\end{array}$ & $\begin{array}{r}-0.3612095 \\
36.9363160 \\
-884.2807841\end{array}$ & $\begin{array}{r}0.0484349 \\
-5.4971981 \\
102.2525869\end{array}$ & $\begin{array}{r}-0.0022475 \\
0.2510974 \\
-2.6516141\end{array}$ \\
\hline
\end{tabular}


Table A.2 Similar to Table A.1, but for stellar populations older than 3.5 Gyr (Age $\geq 3.5 \mathrm{Gyr})$.

\begin{tabular}{|c|c|c|c|c|c|c|}
\hline Index & $j$ & $\mathrm{C}_{1 \mathrm{j}}$ & $\mathrm{C}_{2 \mathrm{j}}$ & $\mathrm{C}_{3 \mathrm{j}}$ & $\mathrm{C}_{4 \mathrm{j}}$ & $\mathrm{C}_{5 \mathrm{j}}$ \\
\hline \multirow{3}{*}{$\mathrm{CN}_{1}$} & 1 & 0.0050070 & -0.0101123 & 0.0016866 & -0.0001101 & 0.0000026 \\
\hline & 2 & -0.7387795 & -0.0268996 & 0.0467357 & -0.0067710 & 0.0002559 \\
\hline & 3 & 1.8158227 & 8.1027523 & -2.3010584 & 0.2598589 & -0.0092777 \\
\hline \multirow{3}{*}{$\mathrm{CN}_{2}$} & 1 & 0.0048267 & -0.0086639 & 0.0014493 & -0.0000926 & 0.0000021 \\
\hline & 2 & -0.6713808 & -0.0767864 & 0.0454875 & -0.0063150 & 0.0002435 \\
\hline & 3 & 2.4347829 & 7.4817292 & -1.9820797 & 0.2264804 & -0.0083295 \\
\hline \multirow{3}{*}{$\mathrm{Ca} 4227$} & 1 & 0.0253654 & -0.0209602 & 0.0048307 & -0.0004936 & 0.0000168 \\
\hline & 2 & -3.5901788 & 1.5319737 & -0.4799447 & 0.0598457 & -0.0022187 \\
\hline & 3 & 108.6370369 & -107.2440863 & 25.4608949 & -2.5477372 & 0.0859891 \\
\hline \multirow{3}{*}{ G4300 } & 1 & 0.2092102 & -0.4271461 & 0.0709524 & -0.0053369 & 0.0001564 \\
\hline & 2 & -32.0561313 & 13.8970486 & -0.9523866 & 0.0227085 & -0.0010081 \\
\hline & 3 & 388.0491119 & -107.1700035 & -9.9303420 & 2.4271319 & -0.0776931 \\
\hline \multirow{3}{*}{ Fe4383 } & 1 & 0.0852039 & -0.2016152 & 0.0323234 & -0.0021218 & 0.0000535 \\
\hline & 2 & -12.6431474 & -2.2748662 & 1.1500142 & -0.1342407 & 0.0045588 \\
\hline & 3 & 60.8341421 & 179.0155270 & -49.0631558 & 5.2647148 & -0.1806644 \\
\hline \multirow{3}{*}{ Ca4455 } & 1 & 0.0439274 & -0.0430992 & 0.0064975 & -0.0004070 & 0.0000095 \\
\hline & 2 & -6.6685854 & 1.4457768 & -0.0535448 & -0.0104817 & 0.0006266 \\
\hline & 3 & 114.6093932 & -5.2478791 & -4.3355245 & 0.8004669 & -0.0341767 \\
\hline \multirow{3}{*}{$\mathrm{Fe} 4531$} & 1 & 0.0586416 & -0.1084756 & 0.0185745 & -0.0013543 & 0.0000372 \\
\hline & 2 & -16.5223730 & 5.9250743 & -0.6822331 & 0.0345448 & -0.0007249 \\
\hline & 3 & 327.8776772 & -131.2118033 & 13.2546833 & -0.3619352 & -0.0008992 \\
\hline \multirow{3}{*}{ Fe4668 } & 1 & 0.0579868 & -0.0883225 & 0.0125230 & -0.0005465 & 0.0000058 \\
\hline & 2 & -14.2549909 & 3.6173449 & -0.0066150 & -0.0775483 & 0.0041877 \\
\hline & 3 & 256.6237954 & 70.1814681 & -29.9674944 & 4.6116691 & -0.1954322 \\
\hline \multirow{3}{*}{$\mathrm{H}_{\beta}$} & 1 & 0.1188078 & 0.1462153 & -0.0301692 & 0.0023434 & -0.0000653 \\
\hline & 2 & 5.2527398 & -5.1845420 & 0.5595370 & -0.0222041 & 0.0004090 \\
\hline & 3 & -6.8462900 & -4.4966224 & 9.4442175 & -1.3358200 & 0.0457839 \\
\hline \multirow{3}{*}{ Fe5015 } & 1 & 0.0327476 & -0.1444852 & 0.0252383 & -0.0018120 & 0.0000483 \\
\hline & 2 & -22.2627396 & 7.4518761 & -0.7875696 & 0.0244834 & 0.0000504 \\
\hline & 3 & 369.7815953 & -72.0823837 & -1.2940653 & 1.1387862 & -0.0545511 \\
\hline \multirow{3}{*}{$\mathrm{Mg}_{1}$} & 1 & -0.0029387 & -0.0005618 & 0.0000571 & -0.0000025 & 0.0000001 \\
\hline & 2 & 0.0681456 & -0.2239214 & 0.0461259 & -0.0037007 & 0.0001017 \\
\hline & 3 & -1.7505218 & 4.5445049 & -1.1536134 & 0.1112986 & -0.0034731 \\
\hline \multirow{3}{*}{$\mathrm{Mg}_{2}$} & 1 & -0.0022995 & -0.0025539 & 0.0005160 & -0.0000415 & 0.0000013 \\
\hline & 2 & -0.3140984 & -0.1102010 & 0.0232351 & -0.0016550 & 0.0000414 \\
\hline & 3 & 3.8491416 & 2.2473119 & -0.6944256 & 0.0739731 & -0.0024430 \\
\hline \multirow{3}{*}{$\mathrm{Mg}_{\mathrm{b}}$} & 1 & 0.0165715 & -0.0215425 & 0.0043674 & -0.0002914 & 0.0000068 \\
\hline & 2 & -8.0187674 & 0.1612416 & 0.0564198 & -0.0091935 & 0.0004596 \\
\hline & 3 & 158.6306124 & -15.8632521 & 0.7547564 & 0.1990004 & -0.0142351 \\
\hline \multirow{3}{*}{ Fe5270 } & 1 & -0.0053182 & -0.0449585 & 0.0074887 & -0.0004761 & 0.0000116 \\
\hline & 2 & -6.2888460 & 0.8351887 & 0.0980695 & -0.0229461 & 0.0009453 \\
\hline & 3 & 106.4680655 & 11.8581491 & -9.0607405 & 1.2154258 & -0.0455243 \\
\hline
\end{tabular}


Table A.2 -continued.

\begin{tabular}{|c|c|c|c|c|c|c|}
\hline Index & $j$ & $\mathrm{C}_{1 \mathrm{j}}$ & $\mathrm{C}_{2 \mathrm{j}}$ & $\mathrm{C}_{3 \mathrm{j}}$ & $\mathrm{C}_{4 \mathrm{j}}$ & $\mathrm{C}_{5 \mathrm{j}}$ \\
\hline \multirow{3}{*}{ Fe5335 } & 1 & -0.0299036 & -0.0168634 & 0.0034824 & -0.0003504 & 0.0000128 \\
\hline & 2 & -0.8764299 & -2.3631112 & 0.3216860 & -0.0034947 & -0.0004859 \\
\hline & 3 & -19.1581245 & 20.3079448 & 1.5735482 & -0.7362636 & 0.0365434 \\
\hline \multirow{3}{*}{ Fe5406 } & 1 & -0.0273466 & -0.0191557 & 0.0032597 & -0.0002321 & 0.0000069 \\
\hline & 2 & -1.6859288 & -1.4089294 & 0.3578634 & -0.0309966 & 0.0008859 \\
\hline & 3 & 3.2750120 & 49.7275784 & -12.4265243 & 1.1493382 & -0.0351427 \\
\hline \multirow{3}{*}{ Fe5709 } & 1 & 0.0058300 & -0.0239914 & 0.0038273 & -0.0002377 & 0.0000055 \\
\hline & 2 & -3.1695351 & 1.3275974 & -0.1282929 & 0.0015248 & 0.0001363 \\
\hline & 3 & 62.3172407 & -5.6986762 & -1.2147039 & 0.3086961 & -0.0142722 \\
\hline \multirow{3}{*}{ Fe5782 } & 1 & -0.0351759 & 0.0072524 & -0.0009035 & -0.0000295 & 0.0000040 \\
\hline & 2 & 2.9123930 & -2.1632509 & 0.2392269 & 0.0009764 & -0.0005652 \\
\hline & 3 & -87.2972968 & 23.4730212 & 0.8426133 & -0.6095760 & 0.0321487 \\
\hline \multirow{3}{*}{$\mathrm{Na}_{\mathrm{D}}$} & 1 & -0.0349510 & -0.0109978 & 0.0032700 & -0.0003007 & 0.0000101 \\
\hline & 2 & -3.1483417 & -0.0799733 & 0.0306158 & 0.0017984 & -0.0001874 \\
\hline & 3 & 74.8532596 & -28.0695426 & 1.8470812 & 0.0045464 & -0.0013129 \\
\hline \multirow{3}{*}{$\mathrm{TiO}_{1}$} & 1 & -0.0019580 & -0.0001078 & 0.0001071 & -0.0000133 & 0.0000005 \\
\hline & 2 & -0.0663381 & 0.0341998 & -0.0108762 & 0.0008305 & -0.0000198 \\
\hline & 3 & 3.2582308 & -1.5575893 & 0.2498990 & -0.0085626 & -0.0000514 \\
\hline \multirow{3}{*}{$\mathrm{TiO}_{2}$} & 1 & -0.0055418 & 0.0002662 & 0.0000839 & -0.0000138 & 0.0000006 \\
\hline & 2 & 0.1861284 & -0.0836849 & 0.0091382 & -0.0007762 & 0.0000260 \\
\hline & 3 & -4.2466215 & 3.0328182 & -0.7327127 & 0.0800970 & -0.0027508 \\
\hline \multirow{3}{*}{$\mathrm{H} \delta_{\mathrm{A}}$} & 1 & -0.4834854 & 0.6747627 & -0.1136504 & 0.0081802 & -0.0002208 \\
\hline & 2 & 48.2162849 & -8.6259083 & -0.5680772 & 0.1330894 & -0.0044290 \\
\hline & 3 & -391.8235480 & -44.3395429 & 41.4350353 & -5.4239881 & 0.1828454 \\
\hline \multirow{3}{*}{$\mathrm{H} \gamma_{\mathrm{A}}$} & 1 & -0.5340026 & 0.8499248 & -0.1407715 & 0.0102053 & -0.0002837 \\
\hline & 2 & 57.5218630 & -12.4432398 & -1.0323349 & 0.2184516 & -0.0069865 \\
\hline & 3 & -381.7703663 & -237.3724815 & 109.5206537 & -12.9300524 & 0.4258356 \\
\hline \multirow{3}{*}{$\mathrm{H} \delta_{\mathrm{F}}$} & 1 & -0.2328216 & 0.3790464 & -0.0645870 & 0.0047630 & -0.0001318 \\
\hline & 2 & 28.4618861 & -8.4343118 & 0.2882612 & 0.0224536 & -0.0007588 \\
\hline & 3 & -256.8427724 & 41.7473144 & 12.0094480 & -2.0067178 & 0.0654486 \\
\hline \multirow{3}{*}{$\mathrm{H} \gamma_{\mathrm{F}}$} & 1 & -0.2081433 & 0.4185126 & -0.0709583 & 0.0052155 & -0.0001461 \\
\hline & 2 & 26.6976093 & -8.4741360 & 0.0074237 & 0.0631139 & -0.0020772 \\
\hline & 3 & -176.6172339 & -82.1781969 & 45.6384532 & -5.4871418 & 0.1779060 \\
\hline
\end{tabular}

